\section{Bilobectomy for allergic bronchopulmonary aspergillosis mimicking lung cancer}

(Scr Med 2011;42:22-5)

\section{Anthony W. Kim \\ David D. Lee ${ }^{2}$ \\ Ozuru O. Ukoha ${ }^{3}$ \\ Marin Sekosan ${ }^{4}$}

\author{
${ }^{1}$ Section of Thoracic Surgery, Yale \\ University, New Haven, CT \\ ${ }^{2}$ Department of Surgery, University of \\ Michigan, Ann Arbor, MI \\ ${ }^{3}$ Division of Cardiothoracic Surgery, \\ John H. Stroger Jr. Hospital of Cook \\ County, Chicago, IL \\ ${ }^{4}$ Department of Pathology, John H. \\ Stroger Jr. Hospital of Cook County, \\ Chicago, IL \\ Correspondence \\ Marin Sekosan, M.D. \\ Chair \\ Division of Anatomic Pathology \\ Stroger Hospital of Cook County \\ 1901 W. Harrison Street \\ Chicago Illinois, 60612 \\ Phone: 312-864-7546 \\ Fax: 312-864-9244 \\ Email:cchmsekosan@yahoo.com
}

Submitted: February 10, 2011

Accepted: March 30, 2011

\begin{abstract}
Allergic Bronchopulmonary Aspergillosis (ABPA) is one of several presentations of pulmonary infections caused by the common fungal organism, Aspergillus fumigatus. ${ }^{1,2}$ ABPA is characterized by colonization of the respiratory tree with the organism in the absence of tissue invasion and allergic reactions, such as Type I immunoglobulin E (IgE)-mediated hypersensitivity and Type III immunoglobulin $\mathrm{G}(\operatorname{IgG})$ immune complex-mediated immune response. ${ }^{1}$ This report presents a patient with a pulmonary mass that was resected due to concerns of malignancy but was ultimately found to be an unusual presentation of allergic bronchopulmonary aspergillosis (ABPA).
\end{abstract}

\section{Case}

The patient is a 54-year-old male with a previous medical history significant only for asthma. He recently ceased tobacco use after smoking approximately less than one-half pack per day for several years. He presented to the emergency department with three weeks of pleuritic chest pain associated with coughing. He denied any other symptoms, including fevers, chills, dyspnea, exertional chest pain, or productive sputum. On physical examination, the only significant finding was mild bilateral wheezing. The remain- der of his physical examination was normal. His white blood cell count was $6,400 / \mathrm{mm}_{3}^{3}$ however, the differential showed an eosinophilia of 0.9 (13.9\%). Additionally, his quantitative IgE antibody count was $1,642 \mathrm{ng} / \mathrm{ml}$. On chest radiography he had a solitary nodule in his right upper lobe. A follow-up computed tomography (CT) scan of his chest demonstrated a mass-like consolidation in the anterior segment of the right upper lobe measuring $6.6 \times 4.3 \mathrm{~cm}$ with patchy areas of consolidation surrounding this area. The consolidation was believed to be post-inflammatory in nature, but an underlying malignancy could not be ruled out. Saccular and central bronchiectasis in the right upper lobe was also noted but was not seen elsewhere in the lungs. (Figure 1)

Flexible bronchoscopy allowed visualization of secretions from the anterior segment bronchus of the right upper lobe (Figure 2). Washings and brushings were negative for malignant cells. Potassium hydroxide $(\mathrm{KOH})$ calcofluor cultures were negative for fungal elements initially and remained negative after six weeks of incubation. The patient was treated with prednisone for over 6 weeks without any change in the size of the mass, as viewed by an interval 
chest CT scan, thus raising a concern for malignancy. Accordingly, he was taken to the operating room for thoracotomy.

The tumor crossed the minor fissure from the anterior segment of the right upper lobe, where the bulk of it was located, to the medial segment of the right middle lobe. No other nodules or masses were palpated. Because an incisional biopsy would have been inappropriate in a malignant setting, a right upper and middle bilobectomy was performed.

The patient's postoperative course was uneventful. Gross pathology revealed ill-defined nodules in the right upper and middle lobes on palpation. These areas formed a poorly circumscribed subpleural and intraparenchymal consolidation measuring approximately $2.0 \times 2.0 \times 2.0 \mathrm{~cm}$. Sections of this area revealed bronchiectasis with mucopurulent material. Mucoid impaction of the bronchi with a large pasty brown plug was noted. Gomori methenamine silver (GMS) staining revealed fungal hyphae in an intrabronchial mucoid plug, but there was no evidence of invasive fungi in the lung parenchyma or bronchial walls. No malignancy was found. (Figure 3)

\section{Discussion}

Allergic Bronchopulmonary Aspergillosis (ABPA) classically presents with symptoms and signs of cough, fever, malaise, anorexia, headache, dyspnea, wheezing, respiratory distress, pleuritic chest pain, hemoptysis, and asthma attacks during episodic or recurrent exacerbations. ${ }^{1,2}$ Clubbing, crackling, and cor pulmonale may be harbingers of end stage disease associated with long standing fibrosis. ${ }^{1,2}$ Pulmonary fibrosis and bronchiectatic changes are believed to develop from inspissated plugs of mucus consisting of aspergillus and eosinophils in the segmental and subsegmental bronchi. ${ }^{2,3}$ Expectoration of these plugs results in the production of green, beige, or brown colored sputum. ${ }^{1}$ During an exacerbation, antigens from proliferating fungi in the airway trigger a type I hypersensitivity reaction with IgE and IgG-mediated release of inflammatory mediators. Necrosis and cellular infiltrates are produced, leaving deposits of immune complexes and inflammatory cells that ultimately cause bronchial wall damage and bronchiectasis. $^{1}$

Eight clinical, radiologic, and serologic criteria have been established to diagnose ABPA: asthma, immediate cutaneous reactivity to Aspergillus, precipitating antibodies to Aspergillus fumigatus, elevated total serum IgE, peripheral eosinophilia, radiologic pulmonary "infiltrates," proximal bronchiectasis, and elevated levels of serum $\operatorname{IgE}$ and IgG compared with those in mold- sensitive asthmatic patients. ${ }^{1}$ The findings of eosinophilia in a setting of asthma or cystic fibrosis should raise the specter of this disease entity, although eosinophilia alone is a nonspecific finding. ${ }^{4}$ If ABPA is suspected, the initial evaluation should include a skin prick test or intradermal reactivity to aspergillus. If this is positive, then the total IgE precipitins to aspergillus can be measured in serum. Elevation of serum IgE, i.e., greater than $1000 \mathrm{ng} / \mathrm{ml}$, can occur in the asthmatic patient, especially during an ABPA exacerbation. A two-fold elevation of IgE Aspergillus fumigatus-specific antibodies are useful for discrimination between asthmatics with and without ABPA. ${ }^{1}$ The real value of total serum IgE is as a clinical tool to track its rise and fall during exacerbations and remissions. ${ }^{1}$

Bronchial impaction with mucous is frequently described as a distinct feature of ABPA. In a study by Bosken and colleagues, 11 of 18 patients with ABPA had mucoid impaction of bronchi. ${ }^{3}$ ABPA has been described on chest radiograph as a transient pulmonary or mass-like infiltrate. The infiltrative appearance is secondary to active or previous mucoid impaction of the proximal bronchi. ${ }^{2,4}$ These mucoid impactions can extend as branching opacities typically in an upper lobe distribution and appear to be associated with central bronchiectasis on plain chest radiography. ${ }^{2}$

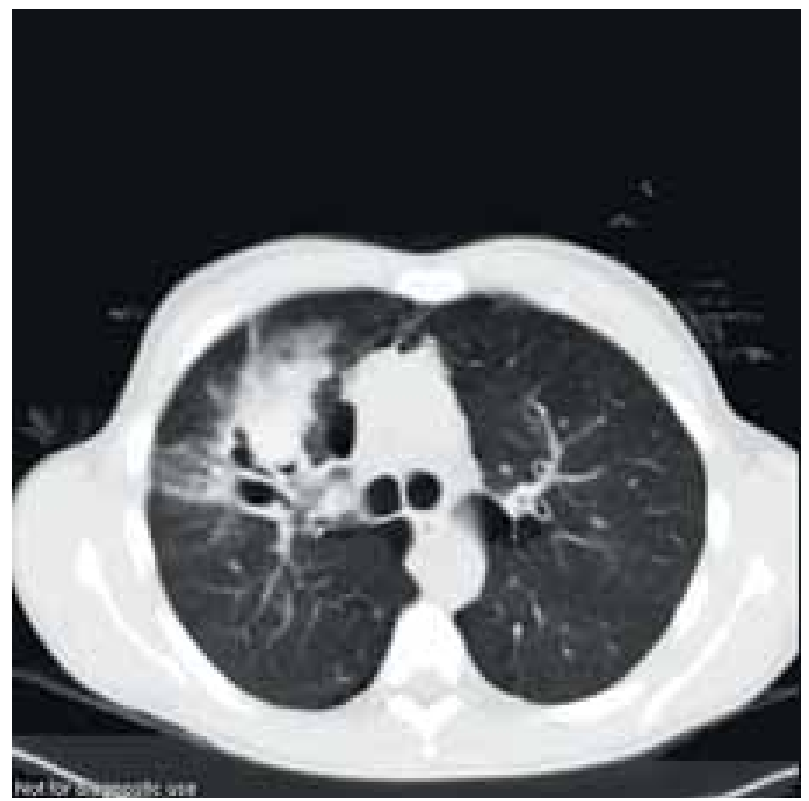

Figure 1. CT scan demonstrating an area of confluence in the anterior segment of the right upper lobe measuring $6.6 \times 4.3$ $\mathrm{cm}$. Patchy areas of consolidation surrounding an area with central bronchiectasis and encroaching on the fissure.

CT scanning is helpful in further delineating ABPA. Bronchial mucus plugging is commonly a characteristic finding in $\mathrm{ABPA}^{4}$ and on CT scan, it can exhibit branching while coursing along the bronchial tree, producing a "finger-like projection," "gloved-finger," or "toothpaste shadow" appearance. The presence of a hyperdense mucus plug may be pathognomonic for ABPA in the setting of asthma. ${ }^{2,4}$ Central bronchiectasis is also strongly suggestive of this disease process, but may be absent early in its course ${ }^{4}$ or present without ABPA in asthmatics. 
PET scanning has not been used routinely for the evaluation of patients with ABPA. It was not used in this case because there was a strong suspicion of malignancy, and PET scanning would be unable to distinguish between neoplasm and infection.

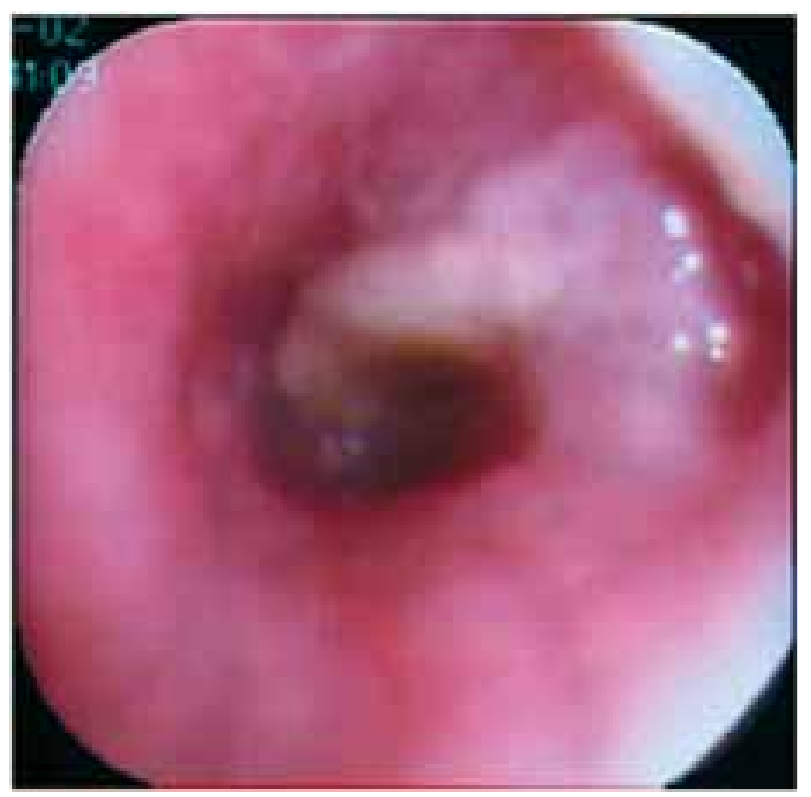

Figure 2. Flexible bronchoscopy revealing minimal secretions form right upper lobe anterior segment bronchus.

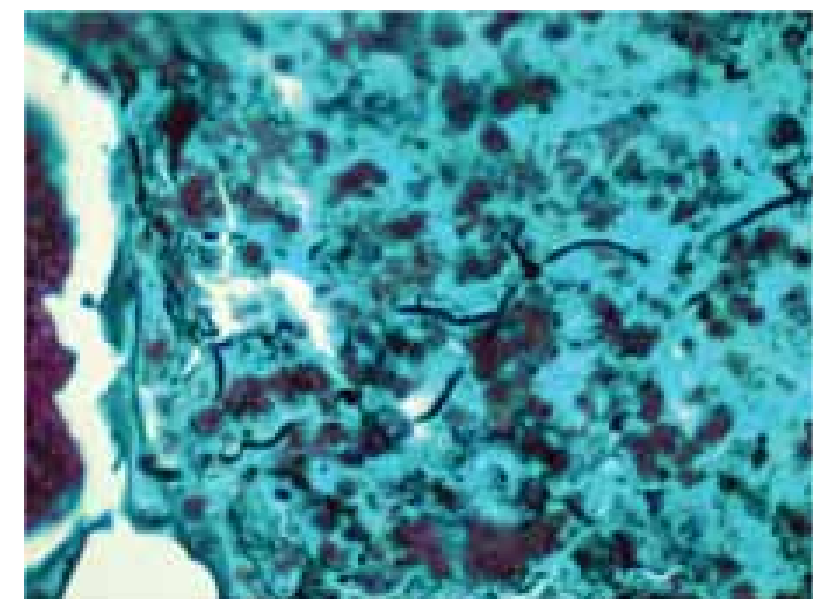

Figure 3. High magnification (450x) of a Gomori Methenamine Silver (GMS) stain slide from an intrabronchial mucoid plug showing rare fungal hyphae morphologically consistent with Aspergillus species.

The presence of ABPA mistaken for a malignancy has been reported. ${ }^{3,5}$ Conversely, the growth pattern of some malignancies have the appearance of and have been mistaken for ABPA. ${ }^{1}$ In a review of ABPA in non-asthmatics, 8 of 19 patients with abnormal radiograph findings of a pulmonary mass were suspected of harboring cancer. ${ }^{5}$ A review of the Western literature indicates that the exact frequency with which ABPA presents as a lung mass is unknown, but it usually is not treated surgically.

Bronchoscopy has been used both in the diagnosis ${ }^{3-5}$ and in the treatment through aspiration ${ }^{4}$ in patients with mucoid impaction. While it may allow for the aspiration of sputum for culture, cultures of Aspergillus fumigatus are not entirely diagnostic and may only provide supportive evidence. ${ }^{1}$ Bronchoscopy findings may be used to rule out malignancy, but the cytology results can be misleading. ${ }^{3,5}$

The patient presented in this report was unique in that the aspergillus infection did not respect the anatomic planes of the lobes of the lung and traversed the minor fissure. Ultimately because of concerns that the mass was malignant, the patient required a bilobectomy, which has not been described previously as a treatment for ABPA. A percutaneous biopsy of the lesion was not performed in the patient reported here, because he had a post-obstructive clinical presentation, which is not uncommon for a malignant neoplasm of the lung. Historically, open lung biopsies were performed to aid in the diagnosis of ABPA. ${ }^{3}$ Because of accurate laboratory tests and improved imaging techniques as well as an improved clinical acumen, the role of open lung biopsy for a relatively straightforward presentation now has decreased utility. A review of the literature suggests that there is no role for routine image-guided percutaneous biopsy of suspected ABPA lesions. ${ }^{1}$ Furthermore, surgical intervention for ABPA is less frequently described; the largest series was reported by Bosken and colleagues. None of their 18 patients underwent a preoperative invasive procedure to arrive at a tissue diagnosis. In particular, five in this cohort did not have enough clinical information to achieve a preoperative diagnosis. ${ }^{3}$

The cornerstone of treatment for ABPA is steroid therapy, which suppresses the immune response. Oral prednisone, slowly tapered over three to six months, is recommended. Clinical responses should be monitored with monthly or bimonthly serum total IgE concentrations. The role of inhaled corticosteroids is undefined. Total serum IgE levels can be monitored to gauge clinical effectiveness along with symptomatic and radiographic improvement. ${ }^{1}$ Once total serum IgE levels normalize, the steroid dosages can be tapered. ${ }^{1}$ Ultimately, steroid therapy results in the resolution of mucoid impaction and bronchiectasis. ${ }^{1}$ The typical rapid resolution of radiographic infiltrates after two to four weeks of therapy should prompt the search for a refractory mucus plug or lung collapse. ${ }^{1}$

While reviews of several studies suggest that antifungal therapy (itraconazole, vorconazole, or other imidazole agents) conveys a modest benefit, its overall usefulness in the treatment of ABPA has yet to be established. Accordingly, unequivocal use of antifungal agents should be limited. ${ }^{1}$ They should be administered as an adjunct to corticosteroid therapy rather than in lieu of it. 
Indications for surgery include: 1 ) persistence of a mass despite of maximal medical therapy in patients with strong risk factors for bronchogenic carcinoma, 2) recurrent or persistent escalating hemoptysis, and 3) post-obstructive complications such as, atelectasis, pneumonia, or lung abscess. The presence of bronchiectasis almost invariably requires anatomic resection rather than wedge resection. The patient presented in this report required a bilobectomy because of the persistence of radiologic abnormalities, concerns for malignancy and the extent of his disease.

\section{Acknowledgements}

The authors acknowledge Eric Cuasay M.D., from the Swedish American Medical Group, Rockford, Illinois for his radiologic expertise.

No potential conflict of interest relevant to this article was reported.

\section{References}

1. Vlahakis NE, Aksamit TR. Diagnosis and treatment of allergic bronchopulmonary aspergillosis. Mayo Clin Proc 2001;76(9):930-8.

2. Gotway MB, Dawn SK, Caoili EM, Reddy GP, Araoz A, Webb RW. The radiologic spectrum of pulmonary aspergillus infections. J Comput Assist Tomogr 2002;26:2:159-73.

3. Bosken CH, Myers JL, Greenberger PA, Katzenstein ALA. Pathologic features of allergic bronchopulmonary aspergillosis. Am J Surg Path 1988;12:216-22.

4. Karunaratne N, Baraket M, Lin S, Ridley L. Thoracic CT illustrating hyperdense bronchial mucous plugging. Australasian Radiology 2003;47:336-8.

5. Sanchez-Alarcos JMF, Martinez-Cruz R, Ortega L, Calle M, Rodriguez-Hermosa JL, Alvarez-Sala JL. ABPA mimicking bronchogenic cancer. Allergy 2001;56:80-1. 